\title{
Nuclear structure dependence of fusion hindrance in heavy element synthesis
}

\author{
J. Khuyagbaatar, ${ }^{1,2,{ }^{*}}$ H. M. David, ${ }^{2}$ D. J. Hinde, ${ }^{3}$ I. P. Carter, ${ }^{3}$ K. J. Cook, ${ }^{3}$ M. Dasgupta, ${ }^{3}$ Ch. E. Düllmann,,${ }^{1,2,4}$ D. Y. Jeung, ${ }^{3}$ \\ B. Kindler, ${ }^{2}$ B. Lommel, ${ }^{2}$ D. H. Luong,,${ }^{3,}$ E. Prasad,${ }^{3,}{ }^{\dagger}$ D. C. Rafferty,${ }^{3}$ C. Sengupta, ${ }^{3}$ C. Simenel, ${ }^{3}$ E. C. Simpson, ${ }^{3}$ \\ J. F. Smith, ${ }^{3,}{ }^{8}$ K. Vo-Phuoc, ${ }^{3}$ J. Walshe, ${ }^{3}$ A. Wakhle, ${ }^{3, \|}$ E. Williams, ${ }^{3}$ and A. Yakushev ${ }^{1,2}$ \\ ${ }^{1}$ Helmholtz Institute Mainz, 55099, Mainz, Germany \\ ${ }^{2}$ GSI Helmholtzzentrum für Schwerionenforschung, 64291, Darmstadt, Germany \\ ${ }^{3}$ Department of Nuclear Physics, RSPE, Australian National University, Canberra, ACT 2601, Australia \\ ${ }^{4}$ Johannes Gutenberg-Universität Mainz, 55099, Mainz, Germany
}

(Received 4 February 2018; revised manuscript received 2 March 2018; published 28 June 2018)

\begin{abstract}
The production of the heaviest elements in fusion-evaporation reactions is substantially limited by very low cross sections, as fusion cross sections (including fusion-fission) are greatly reduced by the competing quasifission mechanism. Using the Australian National University Heavy Ion Accelerator Facility and CUBE detector array, fission fragments from the ${ }^{48} \mathrm{Ti}+{ }^{204,208} \mathrm{~Pb}$ and ${ }^{50} \mathrm{Ti}+{ }^{206,208} \mathrm{~Pb}$ reactions have been measured, with the aim to investigate how the competition between quasifission and fusion-fission evolves with small changes in entrance-channel properties associated mainly with the nuclear structure. Analysis of mass-distribution widths of strongly mass-angle-correlated fission fragments within the framework of the compound-nucleus fission theory demonstrates significant differences in quasifission (and therefore fusion) probabilities among the four reactions. The impact of nuclear structure on fusion highlights the importance of future radioactive beams.
\end{abstract}

DOI: 10.1103/PhysRevC.97.064618

\section{INTRODUCTION}

The fusion of two nuclei is a process relevant for the production of many chemical elements. Moreover, the fusion of heavy $(Z \geqslant 16)$ projectiles and massive $(Z=82-98)$ target nuclei followed by the evaporation of neutrons has been used to synthesize the superheavy elements (SHEs) up to $Z=$ 118 [1-3]. The probability of the fusion-evaporation process proceeding through the formation of a compound nucleus $(\mathrm{CN})$ with full equilibration in all degrees of freedom at an excitation energy $E^{*}$ can be expressed as $P_{f u} W_{\mathrm{CN}}$, where $P_{f u}$ is the fusion probability and $W_{\mathrm{CN}}$ is the survival probability of the $\mathrm{CN}$ through particle evaporation against fission. The fusion can be strongly hindered by the competing quasifission $(\mathrm{QF})$ process, where the two touching nuclei re-separate before reaching equilibrium [4-6]. Generally, QF occurs with shorter sticking times of the two reactant nuclei when compared with reaction timescales for fusion [4-8]. Thus, the properties of fragments from QF are more influenced by dynamical effects associated with the entrance channel (e.g., mass-angle correlation and

\footnotetext{
*J.Khuyagbaatar@gsi.de

${ }^{\dagger}$ Present address: Scandinavian Health Limited, Taiwan Branch, 136 Guosheng 2nd Street, Taoyuan District, Taoyuan City, Taiwan 330.

${ }^{\ddagger}$ Present address: Department of Physics, School of Mathematical and Physical Sciences, Central University of Kerala, Kasaragod 671314, India.

${ }^{\S}$ Present address: Advanced Technology Institute, University of Surrey, Guildford, Surrey, GU2 7XH, United Kingdom.

"Present address: Cyclotron Institute, Texas A\&M University, College Station, Texas 77843, USA.
}

broad mass distribution) compared with those from fusionfission (FF), which are well describable by statistical theories, e.g., through the rotating liquid drop model (RLDM [9]). However, separation, or even quantitative estimates of the contributions from QF and FF are usually limited due to overlap of the experimental observables [4-8,10-18].

Reliable predictions of $\mathrm{P}_{f u}$, which are essential for the successful execution of SHE experiments involving new projectile-target combinations, remain problematic because the QF process is still poorly understood. Consequently, the selection of an appropriate projectile-target combination is one of the important challenges for SHE synthesis.

Fusion hindrance by QF can be studied in reactions leading to formation of the same $\mathrm{CN}$, whose influence can be neglected based on Bohr's independence hypothesis of CN decay [19]. However, reactions with significantly different entrance channels are often used to contrast the fission properties from FF and QF $[5,12,14,20,21]$. Therefore, the effect of a particular variable on fusion hindrance can often not be isolated. To date, the presence of $\mathrm{QF}$ is mainly ascribed to two variables: the product of the projectile and target charge numbers, $Z_{p} Z_{t}$, and the deformation of the target nuclei.

For a long time, $Z_{p} Z_{t}=1600$ was accepted as a lower limit for reactions that have predominantly QF outcomes [22]. Recently, however, evidence for the presence of $\mathrm{QF}$ in reactions with a much smaller $Z_{p} Z_{t}$ has been uncovered $[20,21,23,24]$. In this regard, recent experiments on the ${ }^{34} \mathrm{~S}+$ ${ }^{208} \mathrm{~Pb}$ and ${ }^{36} \mathrm{~S}+{ }^{206} \mathrm{~Pb}$ reactions (which form the same $\mathrm{CN}$ ${ }^{242} \mathrm{Cf}^{*}$, share the same $Z_{p} Z_{t}$, have no static deformation, and are thus expected to have similar $P_{f u} W_{\mathrm{CN}}$ ) have highlighted the effect of nuclear structure on fusion. Fusion-evaporation cross sections of the ${ }^{36} \mathrm{~S}+{ }^{206} \mathrm{~Pb}$ reaction have been found to 
be $\approx 10-10^{2}$ times greater than those of ${ }^{34} \mathrm{~S}+{ }^{208} \mathrm{~Pb}$ over a wide range of $\mathrm{CN}$ excitation energies [23,25]. Based on a series of experimental studies on evaporation-residue and fission cross sections [23,25-27], a higher QF contribution for ${ }^{34} \mathrm{~S}+{ }^{208} \mathrm{~Pb}$ has been proposed to conserve Bohr's hypothesis [19] that $W_{\mathrm{CN}}$ is the same for both reactions.

No evidence for an increased presence of QF in ${ }^{34} \mathrm{~S}+{ }^{208} \mathrm{~Pb}$ has been observed in experimental mass-angle distributions (MADs) of fission fragments [23,26,27]. It has been suggested, therefore, that collisions in the $\mathrm{S}+\mathrm{Pb}$ reactions that result in QF have long sticking times (slow QF), causing a strong overlap between QF and FF observables and rendering any QF dominance in the ${ }^{34} \mathrm{~S}+{ }^{208} \mathrm{~Pb}$ reaction impossible to detect $[23,26,27]$. Thus, experimental signatures of differing fusion probabilities in very similar reactions forming the same $\mathrm{CN}$, which would uniquely resolve these issues, have been urgently sought.

In this work, we search for experimental evidence for differences in QF and FF probabilities between the ${ }^{48} \mathrm{Ti}+$ ${ }^{208} \mathrm{~Pb}$ and ${ }^{50} \mathrm{Ti}+{ }^{206} \mathrm{~Pb}$ reactions, leading to the same $\mathrm{CN}$, ${ }^{256} \mathrm{Rf}^{*}$. These reactions have a higher $Z_{p} Z_{t}=1804$ than that of $\mathrm{S}+\mathrm{Pb}\left(Z_{p} Z_{t}=1312\right)$, resulting in shorter sticking times and consequently a greater contrast between observables of $\mathrm{QF}$ and FF.

\section{EXPERIMENTAL SETUP}

The experiment was carried out at the Heavy Ion Accelerator Facility at the Australian National University (ANU), Canberra. Beams of ${ }^{48} \mathrm{Ti}$ and ${ }^{50} \mathrm{Ti}$ ions were accelerated to energies of 240.0, 245.0, 252.2, 259.0, 270.0, and 280.0 $\mathrm{MeV}$ and 236.0, 240.0, 252.0, 258.0 264.0, 270.0, and $280.2 \mathrm{MeV}$, respectively, by the 14UD electrostatic and the superconducting linear booster accelerators. The beams were delivered in pulses with full widths at half maximum of $\approx 1 \mathrm{~ns}$, separated by $107 \mathrm{~ns}$. The ${ }^{48} \mathrm{Ti}+{ }^{204} \mathrm{~Pb}$ and ${ }^{50} \mathrm{Ti}+{ }^{208} \mathrm{~Pb}$ reactions were also studied because these are expected to have the lowest and highest cross sections for producing $\mathrm{Rf}$ using $\mathrm{Ti}+\mathrm{Pb}$, respectively [1,25,28-31]. Some properties of these reactions are listed in Table I. Lead-sulfide targets with $\approx 100-180 \mu \mathrm{g} / \mathrm{cm}^{2}$ thicknesses (of $\mathrm{Pb}$ ) were used. To minimize any effect of target-thickness inhomogeneities on the measured kinematic quantities of

TABLE I. Summary of reaction properties. Reaction $Q$ values (in $\mathrm{MeV}$, taken from Ref. [33], except the ${ }^{252} \mathrm{Rf}$ mass, which was taken from Ref. [34]), interaction barrier in $\mathrm{MeV}\left(V_{B}\right.$, according to Ref. [35]), entrance-channel mass asymmetries $\left[\alpha=\left(A_{t}-A_{p}\right) /\right.$ $\left.\left(A_{p}+A_{t}\right)\right]$, projectile isospin $\left[T_{z}=(N-Z) / 2\right]$ and the number of nuclear magic numbers in the reactants $\left(N_{m}\right)$ are given.

\begin{tabular}{lcccccc}
\hline \hline Reaction & $\mathrm{CN}$ & $Q$ & $V_{B}$ & $\alpha$ & $T_{z}$ & $N_{m}$ \\
\hline${ }^{48} \mathrm{Ti}+{ }^{204} \mathrm{~Pb}$ & ${ }^{252} \mathrm{Rf}^{*}$ & -165.9 & 196.2 & 0.619 & 2 & 1 \\
${ }^{48} \mathrm{Ti}+{ }^{208} \mathrm{~Pb}$ & ${ }^{256} \mathrm{Rf}^{*}$ & -164.5 & 195.4 & 0.625 & 2 & 2 \\
${ }^{50} \mathrm{Ti}+{ }^{206} \mathrm{~Pb}$ & ${ }^{256} \mathrm{Rf}^{*}$ & -169.4 & 194.6 & 0.609 & 3 & 2 \\
${ }^{50} \mathrm{Ti}+{ }^{208} \mathrm{~Pb}$ & ${ }^{258} \mathrm{Rf}^{*}$ & -169.5 & 194.2 & 0.612 & 3 & 3 \\
${ }^{52} \mathrm{Ti}+{ }^{208} \mathrm{~Pb}$ & ${ }^{260} \mathrm{Rf}^{*}$ & -170.4 & 193.0 & 0.600 & 4 & 2 \\
\hline \hline
\end{tabular}

fission fragments, targets with carbon foils made using two different release agents (betaine and $\mathrm{KCl}$ ) were produced at GSI, Darmstadt, Germany and tested at ANU [32]. Betainecarbon foils, typically used for making large-area targets at GSI, yielded a broad energy distribution of elastically scattered $\mathrm{Ti}$ ions, while the targets made by using $\mathrm{KCl}$ demonstrated much sharper peaks indicating a more uniform thickness [32]. However, a detailed comparison of resultant fission-fragment characteristics measured using the two types of target revealed no significant differences. In the present work the $\mathrm{KCl}$-carbon lead targets were used.

The CUBE detector setup, consisting of two positionsensitive multiwire proportional counters (MWPCs) with dimensions of $28 \times 36 \mathrm{~cm}^{2}$, has recently been expanded by a third, $13 \times 36 \mathrm{~cm}^{2}$ detector. This significantly widens the measurable range of fission-fragment scattering angles. At backward angles, one large and the small MWPC were positioned to face the target perpendicularly such that the center of the large and the edge of the small detector were located 195 and $180 \mathrm{~mm}$ from the target at $90^{\circ}$ and $135^{\circ}$, respectively. The second large MWPC was centered at a $45^{\circ}$ forward angle, $180 \mathrm{~mm}$ from the target center and separated from the two backward detectors by an azimuthal angle of $180^{\circ}$. Data acquisition was triggered by events measured by the forward-angle detector in coincidence with either of the two backward-angle MWPCs. Fission-fragment velocities, scattering angles in the center of mass $\left(\theta_{\text {c.m. }}\right)$, and mass ratios $M_{R}=m_{1} /\left(m_{1}+m_{2}\right)$, where $m_{1}$ and $m_{2}$ are the two fragment masses at scission, were reconstructed by using position and time-of-flight information gathered by the MWPCs [36]. Detailed descriptions of the CUBE in a two-MWPC configuration and data analysis, found in Refs. [6,37,38], can be extended to apply to the three-MWPC system used here. Elastically scattered events were recorded by two silicon surface-barrier detectors mounted at angles of $\pm 30^{\circ}$ on opposite sides of the beam axis to monitor and normalize beam-target interaction.

\section{EXPERIMENTAL RESULTS}

\section{A. Mass ratio and angle distributions}

The experimental distributions of fragment mass ratios and scattering angles in the center of mass of the four reactions can be seen in Fig. 1, measured slightly above, around, and below $V_{B}$ [35]. The MAD matrices are populated by the double-differential cross section $d^{2} \sigma /\left(d \theta_{\text {c.m. }} d M_{R}\right)$. Contributions from scattered projectile and target nuclei are predominantly responsible for the high-intensity regions at $M_{R} \approx 0.2$ and 0.8 , whereas events in the central region originate from fission. The MADs demonstrate a correlation between mass and angle, i.e., slices at particular $\theta_{\text {c.m. }}$ yield different $M_{R}$ centroids. This becomes weaker with decreasing values of $\mathrm{E}_{\mathrm{c} . \mathrm{m} .} / V_{B}$.

Despite the general similarity of the MADs shown in Fig. 1, some differences between different reactions are noticeable. For instance, almost no mass-angle correlation is apparent in either of the ${ }^{50} \mathrm{Ti}$-induced reactions at below-barrier energies (corresponding to the lowest $\mathrm{E}^{*}$ ), leading us to conclude that these fragments may originate from FF, with a possible 


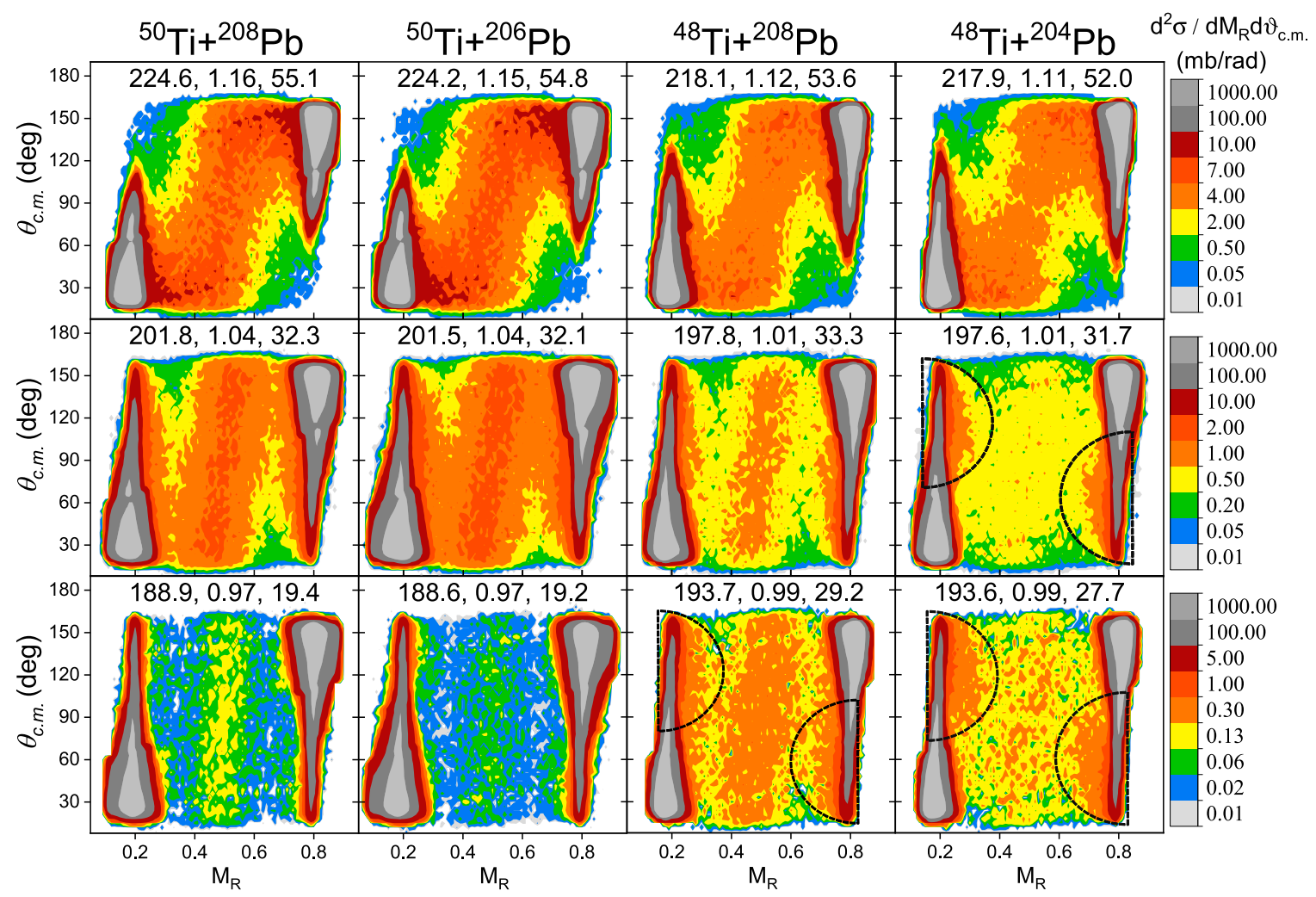

FIG. 1. Measured distributions of mass ratios $\left(M_{R}\right)$ and scattering angles in the center of mass $\left(\theta_{\text {c.m. }}\right)$ for the reactions ${ }^{50} \mathrm{Ti}+{ }^{206,208} \mathrm{~Pb}$ and ${ }^{48} \mathrm{Ti}+{ }^{204,208} \mathrm{~Pb}$. The distributions are labeled by the center-of-mass energy $\left(E_{\mathrm{c} . \mathrm{m} .}, \mathrm{MeV}\right)$, its ratio to interaction barrier $\left(E_{\mathrm{c} . \mathrm{m} .} / V_{B}\right)$, and the excitation energy $\left(E^{*}=E_{\text {c.m. }}-Q, \mathrm{MeV}\right)$. The color palettes were chosen to highlight the region of fission fragments and its numerical levels corresponding to values of double differential cross sections are the same for plots shown by the horizontal axis and are given. Regions marked by dashed lines indicate an appearance of presumably-deep-inelastic reaction products.

contribution from slow QF. In contrast, in both of the ${ }^{48} \mathrm{Ti}$ reactions, relatively broad mass-angle correlations are present at all energies. Closer inspection of these broad MADs reveals, at energies close to or below $V_{B}$, the presence of events close to those corresponding to projectile- and target-like fragments. These events, marked by dashed lines in Fig. 1, were interpreted to originate from deep-inelastic reactions $[4,6,7]$ that proceed on a timescale even shorter than that of QF. Such events are either absent or significantly reduced in the MADs of ${ }^{50} \mathrm{Ti}$ reactions. This may indicate that a change of ${ }^{50} \mathrm{Ti}$ to ${ }^{48} \mathrm{Ti}$ leads to a shortening of interaction times between the colliding nuclei, thus affecting the average sticking time.

\section{B. Mass-ratio distributions}

The mass-ratio distributions of events measured within the range $21^{\circ}<\theta_{\text {c.m. }}<159^{\circ}$ are shown in Fig. 2. In the $M_{R}$ distributions, the fission fragments join smoothly with scattered events and thus do not exhibit a shape describable by a single Gaussian, except the low- $E^{*}$ cases. Nevertheless, distributions exhibit peak-like structures in the region $M_{R} \approx$ $0.35-0.65$. Therefore, one can use the root-mean-square (rms) deviation from $M_{R}=0.5, \sigma_{\mathrm{MR}}$, to quantify the $M_{R}$ distribution of fission fragments [27]. The narrow $M_{R}$ distributions evident in the ${ }^{50} \mathrm{Ti}+{ }^{208} \mathrm{~Pb}$ reaction at $E_{\text {c.m. }} / V_{B} \lesssim 1$ are consistent with the reduced mass-angle correlation and, correspondingly, the smallest QF contribution, i.e., highest fusion, of the reactions measured here. This is supported by the known fact that the fusion-evaporation products of the ${ }^{50} \mathrm{Ti}+{ }^{208} \mathrm{~Pb}$ reaction are observed only at $E_{\mathrm{c} . \mathrm{m} .} / V_{B} \lesssim 1$ with the highest cross-section values among $\mathrm{Ti}+\mathrm{Pb}[1,28-30]$. Likewise, we conclude that the ${ }^{48} \mathrm{Ti}+{ }^{204} \mathrm{~Pb}$ reaction has the largest QF probability. In between these cases lie the ${ }^{48} \mathrm{Ti}+{ }^{208} \mathrm{~Pb}$ and ${ }^{50} \mathrm{Ti}+{ }^{206} \mathrm{~Pb}$ reactions, which have similar widths. The known fusion-evaporation cross sections of the latter reaction are $\approx 10-40$ times greater than the former in the range of $E_{\text {c.m. }}=$ $180-190 \mathrm{MeV}$ [28-30]. However, these data were measured at energies below $V_{B}$, where capture cross sections are strongly affected by the reaction $Q$ value [23] and so cannot directly be used in attributing a higher fusion probability to ${ }^{50} \mathrm{Ti}+{ }^{206} \mathrm{~Pb}$. We note that, at the highest $E^{*}$, the $M_{R}$ distributions of all reactions have similar shapes with larger $\sigma_{\mathrm{MR}}$, indicating a loss of sensitivity to structure effects with increasing $E_{\text {c.m. }}$.

\section{Discussion}

Although the mass-ratio spectra look similar for the ${ }^{48} \mathrm{Ti}+$ ${ }^{208} \mathrm{~Pb}$ and ${ }^{50} \mathrm{Ti}+{ }^{206} \mathrm{~Pb}$ reactions for a wide range of $E^{*}$, the mean square angular momenta $\left(\left\langle J^{2}\right\rangle\right)$ are different. Therefore, to make a qualitative comparison, a baseline dependence of mass-ratio widths on $E^{*}$ and $\left\langle J^{2}\right\rangle$ would be valuable. Effects of angular momenta on the mass-distribution widths are well 


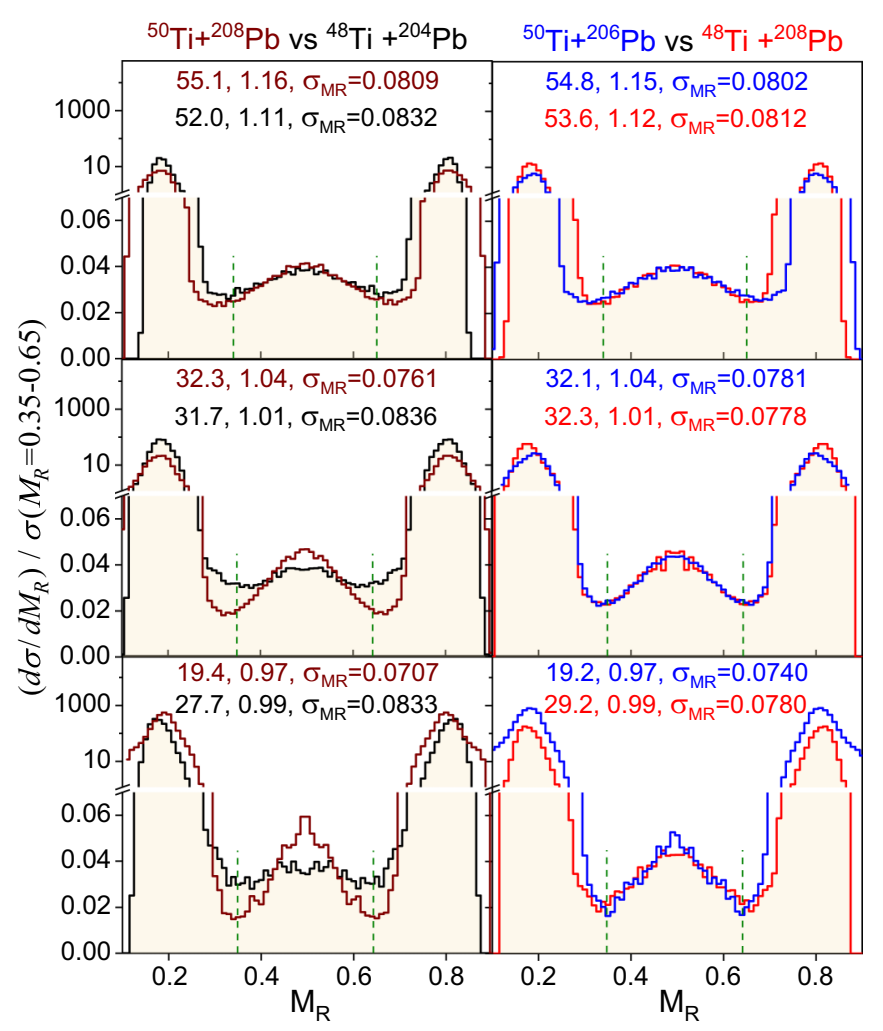

FIG. 2. Mass-ratio distributions of fragments within the range $\theta_{\text {c.m. }}=21^{\circ}-159^{\circ} .{ }^{50} \mathrm{Ti}-$ and ${ }^{48} \mathrm{Ti}$-induced reactions are marked by lined and shaded-lined histograms, respectively. Differential cross sections $\left(d \sigma / d M_{R}\right)$ are normalized by total cross sections integrated over $M_{R}=0.35-0.65$ (marked by dashed lines) where fission is dominant. Excitation energies of the corresponding $\mathrm{CN}$ in $\mathrm{MeV}, \mathrm{E}_{\mathrm{c} . \mathrm{m} .} / V_{B}$ and root-mean-square deviations $\left(\sigma_{\mathrm{MR}}\right)$ within $M_{R}=0.35-0.65$ are given. known [21,27,39-41] and were recently discussed for $\mathrm{S}+\mathrm{Pb}$ reactions where no fast $\mathrm{QF}$ is present, i.e., the MAD shows no mass-angle correlation [27]. Mean square angular momenta $\left\langle J^{2}\right\rangle$ of ${ }^{34} \mathrm{~S}+{ }^{208} \mathrm{~Pb}$ and ${ }^{36} \mathrm{~S}+{ }^{206} \mathrm{~Pb}$ reactions are shown Fig. 3(a). The experimental widths $\sigma_{\mathrm{MR}}(\mathrm{expt})$ (rms deviation) of these reactions measured in the range of $M_{R}=0.25-0.75$ are also shown in Fig. 3(b). The angular momentum $J$ was calculated for each reaction by using the coupled-channels code CCFULL [42], taking into account the appropriate couplings of low-lying excited states [43,44] and deformations [34]. Nuclear potential parameters of $V_{0}=200 \mathrm{MeV}, R_{0}=1.1 \mathrm{fm}$, and $a_{0}=0.75 \mathrm{fm}$ were used.

As expected, at the same $E^{*}$ of the $\mathrm{CN}$, the angular momenta of the two reactions are different, which could cause the observed deviations in their $\sigma_{\mathrm{MR}}$ (expt) [cf. f. 3(a) and 3(b)]. The $\sigma_{\mathrm{MR}}^{2}\left(\right.$ expt) values for ${ }^{34} \mathrm{~S}+{ }^{208} \mathrm{~Pb}$ at $E^{*}>38 \mathrm{MeV}$ can be fit with the well-established semi-empirical expression for the width

$$
\sigma_{\mathrm{MR}}^{2}=C_{T} T+C_{J}\left\langle J^{2}\right\rangle,
$$

where the coefficients $C_{T}=4.54 \times 10^{-3} \mathrm{MeV}^{-1}$ and $C_{J}=$ $1.91 \times 10^{-6} \hbar^{-2}$ were extracted in Ref. [27]. The result of the fit is shown in Fig. 3(b). In Eq. (1), $T$ is the temperature obtained from the $\mathrm{CN} E^{*}$. Temperatures were calculated at saddle points by using the relation

$$
T=\left[\left(E^{*}-B_{f}(J)-E_{\text {rot }}(J)-\left(v_{\text {pre }} 10 \mathrm{MeV}\right) / a\right]^{1 / 2},\right.
$$

where $B_{f}$ and $E_{\text {rot }}$ are the fission barrier and the rotational energy of the excited $\mathrm{CN}$ with angular momentum $J$, respectively. The number of prefission neutrons, $v_{\text {pre }}$, was estimated by using the empirical expression given in Ref. [13], and values of $B_{f}$ and $E_{\text {rot }}$ were taken from RLDM calculations [45]. A level-density parameter $a$ of $A_{\mathrm{CN}} / 10 \mathrm{MeV}^{-1}$ was used.

Note that $\sigma_{\mathrm{MR}}(\mathrm{expt})$ values at $E^{*}<38 \mathrm{MeV}$ were not used for the fit because their corresponding $M_{R}$ distributions have
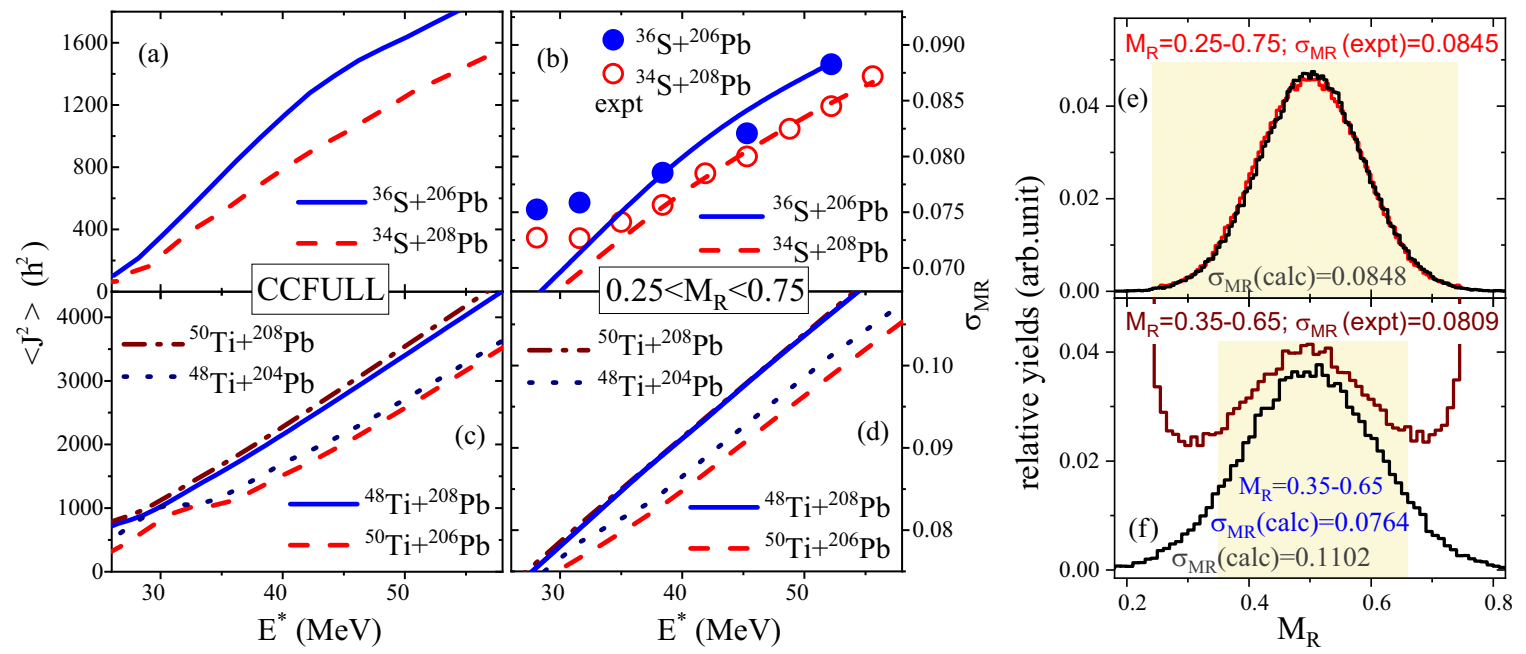

FIG. 3. (a) Calculated mean-squared angular momenta ( $\left\langle J^{2}\right\rangle$, lines) and (b) experimental (symbols) and calculated (lines) widths for $\mathrm{S}+\mathrm{Pb}$ reactions. These data for $\mathrm{S}+\mathrm{Pb}$ were taken from Ref. [27]. (c) Calculated mean-squared angular momenta $\left(\left\langle J^{2}\right\rangle\right)$ and (d) calculated widths for $\mathrm{Ti}+\mathrm{Pb}$ reactions. Legends of symbols and lines are given in panels (a)-(d). Experimental and simulated $M_{R}$ distributions of (e) the ${ }^{34} \mathrm{~S}+{ }^{208} \mathrm{~Pb}$ reaction at $E^{*}=52.2$ and (f) the ${ }^{50} \mathrm{Ti}+{ }^{208} \mathrm{~Pb}$ reaction at $E^{*}=55.1 \mathrm{MeV}$. The experimental $\sigma_{\mathrm{MR}}\left(\right.$ expt) within the appropriate range of $M_{R}$ (shaded region) and the calculated widths $\sigma_{\mathrm{MR}}$ (calc) within the $M_{R}=0.25-0.75$ are given in both panels (e) and (f). rms-deviation of the simulated $M_{R}$ distribution within the $M_{R}=0.35-0.65$ (shaded region) is given in panel (f). See text for detail. 
flat-topped shapes caused by contributions of the multichance fission processes [27].

Expected widths $\sigma_{\mathrm{MR}}$ (calc) for the ${ }^{36} \mathrm{~S}+{ }^{206} \mathrm{~Pb}$ reaction were estimated by Eq. (1) using the coefficients given above, and the results are given in Fig. 3(b). $\left\langle J^{2}\right\rangle$ and $T$ were taken from the results of the CCFULL and Eq. (2), respectively.

As one can see in Fig. 3(b), $\sigma_{\mathrm{MR}}(\mathrm{expt})$ values of the ${ }^{36} \mathrm{~S}+{ }^{206} \mathrm{~Pb}$ reaction are in line with predictions, where $\left\langle J^{2}\right\rangle$ differences of the two reactions were taken into account appropriately in Eq. (1). Accordingly, we suggest a novel viewpoint that the widths of the mass-ratio distributions of the ${ }^{50} \mathrm{Ti}+{ }^{206} \mathrm{~Pb}$ reaction should be larger than those for the ${ }^{48} \mathrm{Ti}+{ }^{208} \mathrm{~Pb}$ reaction at the same $E^{*}$ as, according to the CCFULL calculations shown in Fig. 3(c), $\left\langle J^{2}\right\rangle$ is larger in ${ }^{50} \mathrm{Ti}+{ }^{206} \mathrm{~Pb}$ - if the fast $\mathrm{QF}$ dynamics ( $\sim$ probabilities) are the same! The expected widths for all four $\mathrm{Ti}+\mathrm{Pb}$ reactions calculated in a similar way to the ${ }^{36} \mathrm{~S}+{ }^{206} \mathrm{~Pb}$ reaction are shown in Fig. 3 d). However, these $\sigma_{\text {MR }}($ calc) values cannot directly be compared with the present experimental data because of the narrower $M_{R}=0.35-0.65$ range for the selection of fission-like fragments.

Therefore, to account for any effect on the widths due to the selected $M_{R}$ range, simulated Gaussian distributions with widths from Eq. (1) were randomly generated. The experimental and simulated mass-ratio distributions of fissionlike events for ${ }^{36} \mathrm{~S}+{ }^{208} \mathrm{~Pb}$ and ${ }^{50} \mathrm{Ti}+{ }^{208} \mathrm{~Pb}$ reactions are shown in Figs. 3(e) and 3(f), respectively, to demonstrate the effect of the selected $M_{R}$ range for the rms deviation. The rms deviations within the range $M_{R}=0.35-0.65$ from the simulated distributions [shown in Fig. 3(f) as an example] were then taken as the final values for the predicted widths for the $\mathrm{Ti}+\mathrm{Pb}$ reactions. These results are shown in Fig. 4(a) as a function of $E^{*}$.

The measured mass-ratio widths $\sigma_{\mathrm{MR}}(\mathrm{expt})$ for all four $\mathrm{Ti}+\mathrm{Pb}$ reactions are shown in Fig. $4 \mathrm{~b}$ ). They are all larger than those calculated, indicating in general that the reaction timescale is faster for $\mathrm{Ti}+\mathrm{Pb}$ than for $\mathrm{S}+\mathrm{Pb}$, in agreement with the mass-angle correlations measured here for the former, but not for the latter [27].

To compare quantitatively the measured mass-ratio widths for the $\mathrm{Ti}+\mathrm{Pb}$ reactions, Fig. 4(c) shows the ratio of the experimental to calculated mass-ratio widths $\sigma_{\mathrm{MR}}(\operatorname{expt}) / \sigma_{\mathrm{MR}}(\mathrm{calc})$. This shows a systematic trend from ${ }^{50} \mathrm{Ti}+{ }^{208} \mathrm{~Pb}$ (smallest ratio) to ${ }^{48} \mathrm{Ti}+{ }^{204} \mathrm{~Pb}$ (largest ratio) reactions wherein the smallest and highest contributions of QF, respectively, are attributed. Significantly, the ${ }^{50} \mathrm{Ti}+{ }^{206} \mathrm{~Pb}$ reaction lies below ${ }^{48} \mathrm{Ti}+{ }^{208} \mathrm{~Pb}$. Taking into account the available information regarding fusion-evaporation cross sections for $\mathrm{Ti}+\mathrm{Pb}$ reactions, we therefore conclude that, despite their many similarities (cf. Table I), the contribution from QF is higher for ${ }^{48} \mathrm{Ti}+{ }^{208} \mathrm{~Pb}$ than for ${ }^{50} \mathrm{Ti}+{ }^{206} \mathrm{~Pb}$. This is distinct experimental evidence that different QF probabilities can be seen in reactions leading to the same $\mathrm{CN}$ and having the same $Z_{p} Z_{t}$. Likewise, it confirms the earlier conclusions on the presence of slow $\mathrm{QF}$ in the $\mathrm{S}+\mathrm{Pb}$ reactions made in Refs. [23,26,27].

The entrance channels of reactions studied here are different only due to the structure of the reactants (see Table I). Their structures are reflected in small changes in $V_{B}$ and mass

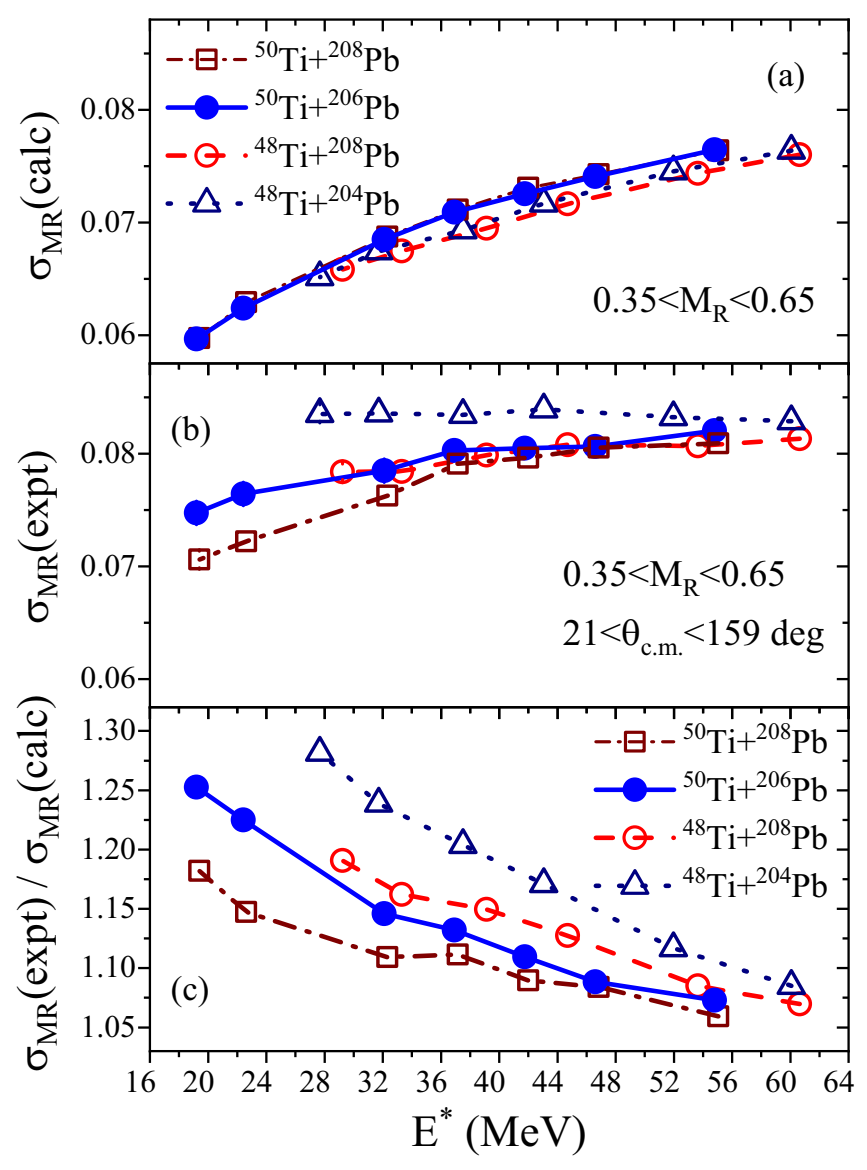

FIG. 4. (a) Calculated and (b) measured rms widths $\left[\sigma_{\mathrm{MR}}(\mathrm{calc})\right.$ and $\left.\sigma_{\mathrm{MR}}(\operatorname{expt})\right]$ of the mass-ratio spectra between $M_{R}=0.35$ and 0.65 . (c) The ratio of calculated and experimental rms widths, $\sigma_{\mathrm{MR}}(\operatorname{expt}) / \sigma_{\mathrm{MR}}(\mathrm{calc})$. Legends of symbols and selected $M_{R}$ range and $\theta_{\text {c.m. }}$ for rms widths are given.

asymmetry $\alpha$, and different $T_{z}$ and $N_{m}$ values, which are responsible for fusion hindrance.

In all reactions, the fusion hindrance (QF) is increasing with an increase of $V_{B}$, which determines the level of Coulomb repulsion at initial touching configurations. Therefore, one can conclude that the Coulomb force, which is typically quantified as $Z_{p} Z_{t}$, is still the predominant factor for hindering fusion. At the touching configuration, the other variables associated with the nuclear structure of reactants (e.g., $T_{z}$ and $N_{m}$ ) may also play important roles.

An increase of $\mathrm{QF}$ when a given projectile (with the same $T_{z}$ ) is paired with a lighter-mass target is observed. This is evident in Fig. 4(c), where $\sigma_{\mathrm{MR}}($ expt $) / \sigma_{\mathrm{MR}}\left(\right.$ calc) is larger for ${ }^{50} \mathrm{Ti}+$ ${ }^{206} \mathrm{~Pb}$ than for ${ }^{50} \mathrm{Ti}+{ }^{208} \mathrm{~Pb}$. Similarly, for the ${ }^{48} \mathrm{Ti}$ reactions, deviations of widths measured using ${ }^{204} \mathrm{~Pb}$ are greater than those involving ${ }^{208} \mathrm{~Pb}$. Therefore, in reactions with the same $T_{z}$, more mass-asymmetric reactants could be favorable for fusion.

Note that $N_{m}$ could have a stronger impact on the QF than $\alpha$. A higher level of $\mathrm{QF}$ in ${ }^{48} \mathrm{Ti}+{ }^{204} \mathrm{~Pb}\left(N_{m}=1\right)$ than in ${ }^{50} \mathrm{Ti}+{ }^{208} \mathrm{~Pb}\left(N_{m}=3\right)$ supports the supposition made in Refs. [46,47] that an increased $N_{m}$ enhances $P_{f u}$. However, our results demonstrate that two reactions, leading to the same 
CN with the same $N_{m}=2$, exhibit differing QF contributions. This may lead to the conclusion that the presence of $N_{m}$ in both the projectile and target nuclei (e.g., ${ }^{50} \mathrm{Ti}+{ }^{206} \mathrm{~Pb}$ ) can strengthen the fusion enhancement. Clearly, the influence of $N_{m}$ on fusion must be further explored. This is of particular importance for the synthesis of SHE beyond $\mathrm{Og}(Z=118)$, where reactions with at least one magic number less than those using ${ }^{48} \mathrm{Ca}$ as a projectile must be employed $[5,48]$.

It is a known fact that reactions involving more neutron-rich projectile nuclei (where $T_{z}$ are high) have been typically found to demonstrate the highest fusion-evaporation cross sections [1]. The observed QF dominance in ${ }^{48} \mathrm{Ti}$ reactions (where $T_{z}$ is smaller than for ${ }^{50} \mathrm{Ti}$ ) supports the impact of neutron richness on fusion [49]. Moreover, the present data are valuable when considering the importance of radioactive-ion beams. With this in mind, we consider ${ }^{52} \mathrm{Ti}$ as a possible projectile, paired with ${ }^{208} \mathrm{~Pb}$ (see Table I). This reaction, with the smallest $V_{B}$ and the highest $T_{z}=4$ compared with the four reactions explored here, and $N_{m}=2$, could result in significant fusion enhancement compared with ${ }^{50} \mathrm{Ti}+{ }^{208} \mathrm{~Pb}$.

\section{SUMMARY AND CONCLUSION}

The results presented here demonstrate a significant variation of fusion probabilities with very small changes in entrance channel for reactions with the same $Z_{p} Z_{t}$ that cannot be explained in current models. These effects could be associated with the structure of the colliding nuclei, thus indicating a promising future for the application of radioactive-ion beams. Considering that $\mathrm{QF}$ can occur on a wide range of reaction timescales, originating from the decay of nuclear matter with partially equilibrated degrees of freedom, current knowledge on the QF process accumulated from experimental observables could be lacking critical aspects as many important properties of QF may remain hidden through the overlap of slow QF with FF properties.

\section{ACKNOWLEDGMENTS}

We are indebted to Dr. N. Lobanov, Dr. T. Kibedi, and the staff of the ANU Heavy Ion Accelerator Facility for their assistance with accelerator operations. J. Steiner and V. Yakusheva are acknowledged for target preparations. The authors acknowledge the support of Australian Research Council research grants DE140100784, DP140101337, DP160101254, DP170102318, DP170102423, German Academic Exchange Service (DAAD) via funds of the German Federal Ministry of Education and Research (BMBF). The Australian Federal Government NCRIS program is acknowledged for support of operations of the ANU Heavy Ion Accelerator Facility.
[1] S. Hofmann and G. Münzenberg, Rev. Mod. Phys. 72, 733 (2000).

[2] Yu. T. Oganessian and V. K. Utyonkov, Nucl. Phys. A 944, 62 (2015).

[3] K. Morita et al., J. Phys. Soc. Jpn. 81, 103201 (2012).

[4] J. Toke et al., Nucl. Phys. A 440, 327 (1985).

[5] M. G. Itkis et al., Nucl. Phys. A 787, 150 (2007).

[6] R. du Rietz, E. Williams, D. J. Hinde, M. Dasgupta, M. Evers, C. J. Lin, D. H. Luong, C. Simenel, and A. Wakhle, Phys. Rev. C 88, 054618 (2013).

[7] R. Bock et al., Nucl. Phys. A 388, 334 (1982).

[8] W. Q. Shen, J. Albinski, A. Gobbi, S. Gralla, K. D. Hildenbrand, N. Herrmann, J. Kuzminski, W. F. J. Müller, H. Stelzer, J. Toke, B. B. Back, S. Bjornholm, and S. P. Sorensen, Phys. Rev. C 36, 115 (1987).

[9] S. Cohen, F. Plasil, and W. J. Swiatecki, Ann. Phys. (NY) 82, 557 (1974).

[10] M. B. Tsang, D. Ardouin, C. K. Gelbke, W. G. Lynch, Z. R. $\mathrm{Xu}$, B. B. Back, R. Betts, S. Saini, P. A. Baisden, and M. A. McMahan, Phys. Rev. C 28, 747 (1983).

[11] B. B. Back, R. R. Betts, J. E. Gindler, B. D. Wilkins, S. Saini, M. B. Tsang, C. K. Gelbke, W. G. Lynch, M. A. McMahan, and P. A. Baisden, Phys. Rev. C 32, 195 (1985).

[12] B. B. Back, P. B. Fernandez, B. G. Glagola, D. Henderson, S. Kaufman, J. G. Keller, S. J. Sanders, F. Videbæk, T. F. Wang, and B. D. Wilkins, Phys. Rev. C 53, 1734 (1996).

[13] M. G. Itkis and A. Ya. Rusanov, Phys. Part. Nucl. 29, 160 (1998).

[14] D. J. Hinde, M. Dasgupta, and A. Mukherjee, Phys. Rev. Lett. 89, 282701 (2002).

[15] M. G. Itkis et al., Nucl. Phys. A 834, 374c (2010).

[16] D. J. Hinde, R. G. Thomas, R. du Rietz, A. Diaz-Torres, M. Dasgupta, M. L. Brown, M. Evers, L. R. Gasques, R. Rafiei, and M. D. Rodriguez, Phys. Rev. Lett. 100, 202701 (2008).
[17] E. M. Kozulin et al., Phys. Lett. B 686, 227 (2010).

[18] K. Nishio, S. Hofmann, F. P. Heßberger, D. Ackermann, S. Antalic, Y. Aritomo, V. F. Comas, C. E. Düllmann, A. Gorshkov, R. Graeger, K. Hagino, S. Heinz, J. A. Heredia, K. Hirose, H. Ikezoe, J. Khuyagbaatar, B. Kindler, I. Kojouharov, B. Lommel, R. Mann, S. Mitsuoka, Y. Nagame, I. Nishinaka, T. Ohtsuki, A. G. Popeko, S. Saro, M. Schädel, A. Türler, Y. Watanabe, A. Yakushev, and A. V. Yeremin, Phys. Rev. C 82, 024611 (2010).

[19] N. Bohr, Nature (London) 137, 344 (1936).

[20] A. C. Berriman et al., Nature (London) 413, 144 (2001).

[21] G. N. Knyazheva, E. M. Kozulin, R. N. Sagaidak, A. Y. Chizhov, M. G. Itkis, N. A. Kondratiev, V. M. Voskressensky, A. M. Stefanini, B. R. Behera, L. Corradi, E. Fioretto, A. Gadea, A. Latina, S. Szilner, M. Trotta, S. Beghini, G. Montagnoli, F. Scarlassara, F. Haas, N. Rowley, P. R. S. Gomes, and A. SzantodeToledo, Phys. Rev. C 75, 064602 (2007).

[22] W. J. Swiatecki, Phys. Scr. 24, 113 (1981).

[23] J. Khuyagbaatar, K. Nishio, S. Hofmann, D. Ackermann, M. Block, S. Heinz, F. P. Hessberger, K. Hirose, H. Ikezoe, B. Kinlder, B. Lommel, H. Makii, S. Mitsuoka, I. Nishinaka, T. Ohtsuki, Y. Wakabayashi, and S. Yan, Phys. Rev. C 86, 064602 (2012).

[24] D. J. Hinde and M. Dasgupta, Phys. Lett. B 622, 23 (2005).

[25] J. Khuyagbaatar et al., Eur. Phys. J. A 46, 59 (2010).

[26] J. Khuyagbaatar et al., EPJ Web Conf. 63, 02015 (2013).

[27] J. Khuyagbaatar, D. J. Hinde, I. P. Carter, M. Dasgupta, C. E. Düllmann, M. Evers, D. H. Luong, R. du Rietz, A. Wakhle, E. Williams, and A. Yakushev, Phys. Rev. C 91, 054608 (2015).

[28] I. Dragojević, K. E. Gregorich, C. E. Dullmann, M. A. Garcia, J. M. Gates, S. L. Nelson, L. Stavsetra, R. Sudowe, and H. Nitsche, Phys. Rev. C 78, 024605 (2008).

[29] F. P. Hessberger et al., Z. Phys. A: Hadrons Nucl. 359, 415 (1997) 
[30] J. Khuygbaatar et al., GSI Sci. Rep. 2012, 133 (2013).

[31] J. Khuyagbaatar et al., Eur. Phys. J. A 37, 177 (2008).

[32] A. Hübner et al., AIP Conf. Proc. 1962, 030001 (2018).

[33] M. Wang et al., Chin. Phys. C 36, 1603 (2012).

[34] P. Möller et al., At. Data Nucl. Data Tables 59, 185 (1995).

[35] R. Bass, Phys. Rev. Lett. 39, 265 (1977).

[36] D. J. Hinde, M. Dasgupta, J. R. Leigh, J. C. Mein, C. R. Morton, J. O. Newton, and H. Timmers, Phys. Rev. C 53, 1290 (1996).

[37] R. Rafiei, R. G. Thomas, D. J. Hinde, M. Dasgupta, C. R. Morton, L. R. Gasques, M. L. Brown, and M. D. Rodriguez, Phys. Rev. C 77, 024606 (2008).

[38] R. G. Thomas, D. J. Hinde, D. Duniec, F. Zenke, M. Dasgupta, M. L. Brown, M. Evers, L. R. Gasques, M. D. Rodriguez, and A. Diaz-Torres, Phys. Rev. C 77, 034610 (2008).

[39] C. Lebrun et al., Nucl. Phys. A 321, 207 (1979).

[40] E. G. Ryabov, A. V. Karpov, and G. D. Adeev, Nucl. Phys. A 765, 39 (2006).
[41] C. J. Lin, R. du Rietz, D. J. Hinde, M. Dasgupta, R. G. Thomas, M. L. Brown, M. Evers, L. R. Gasques, and M. D. Rodriguez, Phys. Rev. C 85, 014611 (2012).

[42] K. Hagino et al., Comput. Phys. Commun. 123, 143 (1999).

[43] S. Raman et al., At. Data Nucl. Data Tables 36, 1 (1987).

[44] R. H. Spear, At. Data Nucl. Data Tables 42, 55 (1989).

[45] A. J. Sierk, Phys. Rev. C 33, 2039 (1986).

[46] C. Simenel et al., Phys. Lett. B 710, 607 (2012).

[47] G. Mohanto, D. J. Hinde, K. Banerjee, M. Dasgupta, D. Y. Jeung, C. Simenel, E. C. Simpson, A. Wakhle, E. Williams, I. P. Carter, K. J. Cook, D. H. Luong, C. S. Palshetkar, and D. C. Rafferty, Phys. Rev. C 97, 054603 (2018).

[48] J. Khuyagbaatar, EPJ Web Conf. 163, 00030 (2017).

[49] K. Hammerton, Z. Kohley, D. J. Hinde, M. Dasgupta, A. Wakhle, E. Williams, V. E. Oberacker, A. S. Umar, I. P. Carter, K. J. Cook, J. Greene, D. Y. Jeung, D. H. Luong, S. D. McNeil, C. S. Palshetkar, D. C. Rafferty, C. Simenel, and K. Stiefel, Phys. Rev. C 91, 041602(R) (2015). 